\title{
Effects of Humidity on the Interaction between a Fused Silica Test Mass and an Electrostatic Drive
}

\author{
Koptsov D.V.*, Prokhorov L.G., Mitrofanov V.P. \\ Faculty of Physics, M.V. Lomonosov Moscow State University, 119991, Moscow, Russia
}

\begin{abstract}
Interaction of a fused silica test mass with electric field of an electrostatic drive with interdigitated electrodes and influence of ambient air humidity on this interaction are investigated. The key element of the experimental setup is the fused silica torsional oscillator. Time dependent increase of the torque acting on the oscillator's plate after application of DC voltage to the drive is demonstrated. The torque relaxation is presumably caused by the redistribution of electric charges on the fused silica plate. The numerical model has been developed to compute the time evolution of the plate's surface charge distribution and the corresponding torque.
\end{abstract}

Keywords: electrostatic drive, dielectric surface conductivity, charges accumulation, Poisson-Nernst-Planck equations, relaxation

\section{Introduction}

Investigation of dielectric charging and transport of electric charges in dielectrics has a long history. Firstly, the interest was caused by a struggle with static electrification and its negative effects in industry, then by the development 5 of various electret devices. In recent years, the problem of the time-dependent dielectric charging in radio frequency micromechanical system (MEMS) capacitive switches as well as in electrostatically actuated MEMS micromirrors is attracting more attention because the charging has significant impact on the device performance and leads to reduction of the reliability and the lifetime of 10 these devices [1]3. Electrostatic actuators are also used for control of dielectric mirrors in sensitive laser interferometers and advanced gravitational wave detectors [4, 5]. The characteristic time of charge accumulation and charge decay can reach several years for materials with excellent insulating properties, such as fused silica $\mathrm{SiO}_{2}$, placed in vacuum [6, 7]. While bulk charging is not ex-

15 pected to be sensitive to environmental conditions, surface charging and surface

\footnotetext{
${ }^{*}$ Corresponding author

Email address: kopcov@physics.msu.ru (Koptsov D.V.)
}

Preprint submitted to Physics Letters A

May 21, 2015

(C) 2015. This manuscript version is made available under the Elsevier user license http://www.elsevier.com/open-access/userlicense/1.0/ 


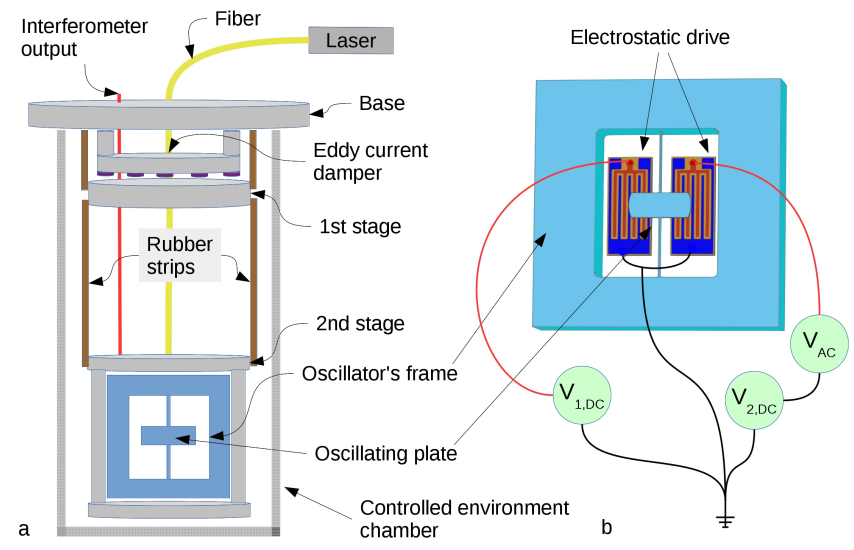

Figure 1: Schematic of the experimental setup. a) Double pendulum suspension of the frame with the oscillator. b) Electrostatic drives with voltage application scheme.

electrical conduction are affected by ambient humidity [8. Study of dielectric charging under different relative humidity levels allows deeper understanding of this process. Various experimental techniques including Kelvin probe force microscopy, measurement of the capacitance-voltage characteristic, discharge 20 current transient method and other are employed to study dielectric charging phenomena and surface conduction [8 10 .

In this paper, we present results of experimental investigations of time dependent response of a fused silica torsional mechanical oscillator to a step-like voltage applied to the nearby electrostatic drive (ESD) with interdigitated elec-

25 trodes. This transient is associated with redistribution and accumulation of electric charges on the fused silica plate of the oscillator. The charge accumulates in peaks mirroring the electrode pattern. Measurements were carried out under different relative humidity levels. Finite element simulation of charge migration on the surface of the plate and their interaction with the electrostatic 30 drive has shown a good agreement with the observed behavior of the oscillator.

\section{Experimental setup}

The experimental setup, specially developed to investigate the interaction between fused silica plate and the ESD is presented in Fig. 1. The fused silica test mass is a rectangular plate $\left(25.4 \times 10 \times 2.5 \mathrm{~mm}^{3}\right)$ welded via thin fused

35 silica fibers to a rectangular frame so that a monolithic torsional oscillator is formed. Two FR4 glass epoxy laminate plates with gold coated interdigitated electrodes form two electrostatic drives. They are placed parallel to the plate of the oscillator at a distance of about $1.5 \mathrm{~mm}$. The interdigitated electrodes are formed by an array of strips $1 \mathrm{~mm}$ wide with period of $2 \mathrm{~mm}$ alternatively at

40 the electric potentials $U_{D C / A C}$ and 0 (grounded). Inhomogeneous electric field created by the electrodes of the ESD produces the electrostatic force acting on 
the dielectric plate. Each ESD is mounted on an underlying aluminum baseplate and has an independent voltage source so that $\mathrm{DC}, \mathrm{AC}$ or $\mathrm{DC}+\mathrm{AC}$ voltages can be applied to it. The ESD's baseplates can be moved to adjust the distance

45 between each ESD and the oscillator's plate in order to compensate the action of the ESDs when DC voltage is applied to them and thus reduce the twist of the oscillator's plate. Further fine tuning is achieved by slightly varying the difference between DC voltages applied to the ESDs. The oscillator and the ESDs are surrounded by grounded metal parts that form an electrostatic

50 shield. The torsional oscillator's resonant frequency $f_{0}=63.13 \mathrm{~Hz}$, the quality factor $Q \approx 10^{6}$ in vacuum and $Q \approx 2100$ in air. The twist angle is measured with a specially designed Michelson interferometer. The back surface of the oscillator's plate has a reflective dielectric coating. It forms two moving in counter-phase mirrors of this interferometer [11. The oscillator, the ESD plates 55 and the interferometer are mounted on a slab that is suspended as a double pendulum by rubber strips. Each stage is suspended using 3 strips with sizes of $20 \times 4 \times 80 \mathrm{~mm}^{3}$ for the upper stage and $20 \times 4 \times 200 \mathrm{~mm}^{3}$ for the lower stage. The upper stage of the double pendulum is damped by means of an eddy current damper in order to provide seismic isolation. The system is placed in

60 the humidity controlled chamber. The sensitivity of the experimental setup at frequencies near the resonant frequency of the oscillator is found to be close to the the limit determined by the torque thermal fluctuations with power spectral density $S_{M}(\omega) \approx 10^{-28}(\mathrm{Nm})^{2} / \mathrm{Hz}$.

Measurements were carried out at room temperature at different relative 65 humidity levels $h(30 \% \leqslant R H \leqslant 60 \%)$. Before each measurement the sample was kept in the closed chamber for a day or more in order to achieve equilibrium between humid air and the sample. The absence of air breakdown was controlled by monitoring the noise spectrum of the torque acting on the oscillator.

The electrostatic force exerted by the ESD on the fused silica plate produces

70 a torque $M$ turning the plate. It can be related to the voltage $U$ applied to the electrodes [12]:

$$
M_{1}=\frac{1}{2} \frac{d C}{d \theta} U^{2}=A U^{2},
$$

where $C$ is the capacitance between the electrodes at potential $U$ and grounded electrodes of the ESD, $\theta$ is the oscillator's plate twist angle, $A$ is the constant of proportionality.

75 An additional torque is produced if the fused silica plate of the oscillator is charged. It can be written as 13 :

$$
M_{2}=B U+M_{i m},
$$

where the constant of proportionality $B$ and $M_{i m}$ depend on the amount and distribution of the charge that is present on the plate as well as on the geometry of the system, i.e. position of the ESD, the plate, surrounding metal and dielec-

so tric objects. The term $M_{i m}$ is associated with the electrostatic image force and is considered negligible in the further analysis. Redistribution and accumulation 


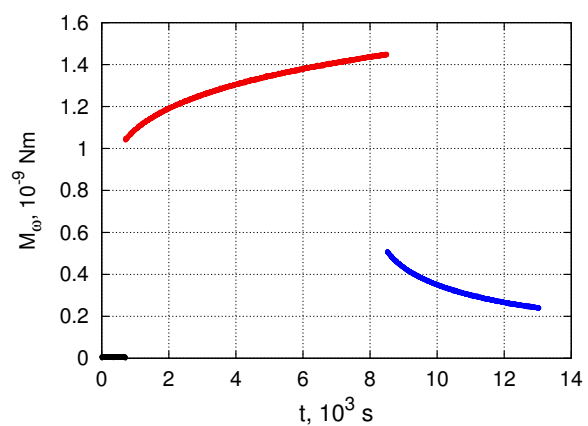

Figure 2: Time evolution of the torque $M_{\omega}(t)$ before $U_{D C}$ is applied $(t=0-700 \mathrm{~s}$, black line), during the application of $U_{D C}\left(t=700-8500 \mathrm{~s}\right.$, red line) and after $U_{D C}$ is switched off $(t>8500 \mathrm{~s}$, blue line).

of electric charges on the fused silica plate of the oscillator alters the second term of the full torque $M=M_{1}+M_{2}$. In the experiment, AC voltage $\left(U_{A C} \cos \omega t\right)$ with $U_{A C}=0-20 \mathrm{~V}$ and DC voltage $U_{D C}=0-1000 \mathrm{~V}$ are applied to the electrodes of the ESD, and the amplitude of the twist angle $\theta_{\omega}$ of the oscillator's plate at the frequency $\omega=2 \pi 36 \mathrm{~Hz}$ is monitored. The amplitude of the torque $M_{\omega}=M_{\omega, 1}+M_{\omega, 2}$ at the frequency $\omega$ is calculated using the relationship

$$
\theta_{\omega}=K(\omega) M_{\omega},
$$

where $K(\omega)$ is a modulus of mechanical susceptibility of the torsional oscillator which describes its angular response $\theta_{\omega}$ to the torque $M_{\omega}$ at the frequency $\omega$ :

$$
K(\omega)^{-1}=J\left(\left(\omega^{2}-\omega_{0}^{2}\right)^{2}+\omega^{2} \omega_{0}^{2} / Q^{2}\right)^{1 / 2},
$$

where $J$ - moment of inertia of the torsional oscillator's plate, $\omega_{0}=2 \pi f_{0}$ resonant angular frequency, $Q$ - quality factor.

In the experiment, $U_{D C}$ is applied to both ESDs in order to compensate their action. $U_{A C}$ is applied only to the first ESD and produces the torque with the amplitude

$$
M_{\omega}=2 A U_{D C} U_{A C}+B U_{A C} .
$$

A change of the charge distribution on the fused silica plate changes of the coefficient $B$ and therefore the torque $M_{\omega}$.

\section{Results of measurements}

The constant of proportionality $A$ in Eq. 1 can be determined from the dependence of the amplitude of the torque $M_{\omega}$ on $U_{A C}$. If the charge on the surface of the oscillator's plate is small, i.e. $M_{\omega, 1} \gg M_{\omega, 2}$,

$$
M_{\omega} \approx 2 A U_{D C} U_{A C}
$$




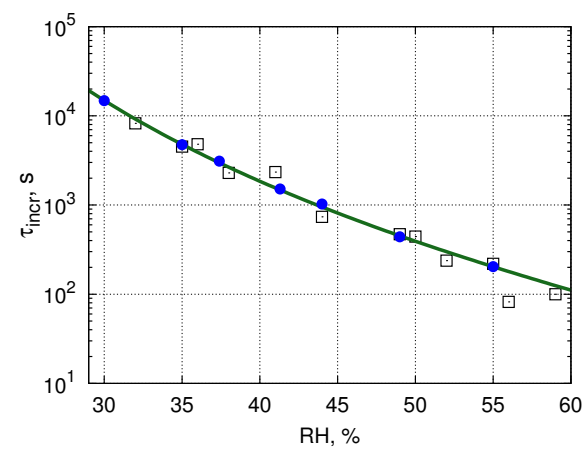

(a)

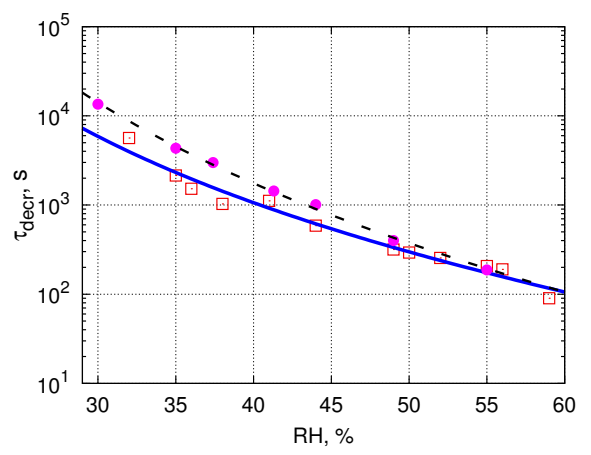

(b)

Figure 3: Time constants of the process of torque $M_{\omega}$ increase (a) and decrease (b) plotted as function of relative humidity level; experimental data are shown with squares, numerical simulation data with circles, solid and dashed lines are respectively the experimental and numerical data fits with a function similar to $\mathrm{Eq} 8$

and $A$ can be determined from the measurement of $M_{\omega}$ as a function of $U_{A C}$ provided that $U_{D C}$ is constant. The value of $A$ was found to be $\approx 2.5 \cdot 10^{-14} \mathrm{Nm} / \mathrm{V}^{2}$.

The time evolution of the torque $M_{\omega}(t)$ calculated from the measured amplitude of the forced oscillations at the frequency $\omega=2 \pi 36 \mathrm{~Hz}$ according to Eq. 3 is shown in Fig. 2. $U_{A C}$ is applied to one of the ESDs during all the time at all stages of the measurement. At the first stage $U_{D C}$ is switched off in order 105 to measure the initial level of the torque due to preexisting charge distribution. At the next stage $U_{D C}$ is applied to both ESDs. At the 3rd stage the $U_{D C}$ is switched off.

After application of the DC voltage one can see a fast increase of $M_{\omega}$ associated with a term $2 A U_{D C} U_{A C}$ and a subsequent slow increase of $M_{\omega}$. After DC voltage is turned off and the electrodes of the ESDs are grounded a fast decrease of $M_{\omega}$ and its subsequent slow decrease are observed. A time-dependent increase of $M_{\omega}$ ("incr") and a time-dependent decrease ("decr") can be fitted using the damped least-squares method. The stretched exponential function has been chosen because it is often used to describe relaxation processes in disordered systems [14, in particular charge decay processes in dielectrics [15]:

$$
\begin{aligned}
M_{\omega, \text { incr }}(t)= & M_{\omega, \text { incr }}(t=\infty)- \\
& \Delta M_{\text {incr }} \exp \left(-\left(t / \tau_{\text {incr }}\right)^{\beta_{\text {incr }}}\right), \\
M_{\omega, \text { decr }}(t)= & M_{\omega, 0}+ \\
\Delta & M_{\text {decr }} \exp \left(-\left(\frac{t-t_{\text {off }}}{\tau_{\text {decr }}}\right)^{\beta_{\text {decr }}}\right),
\end{aligned}
$$

where $M_{\omega, 0}$ is the initial level of the torque associated with the preexisting 


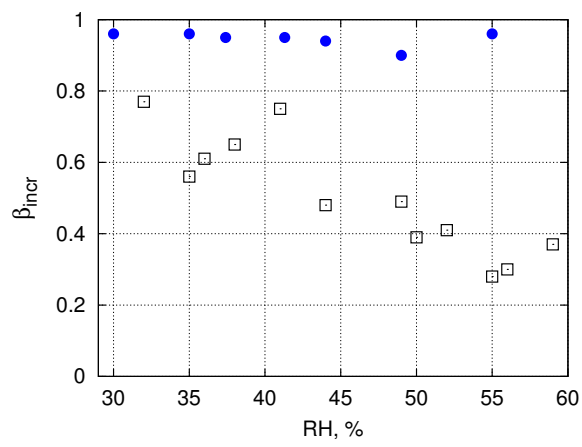

(a)

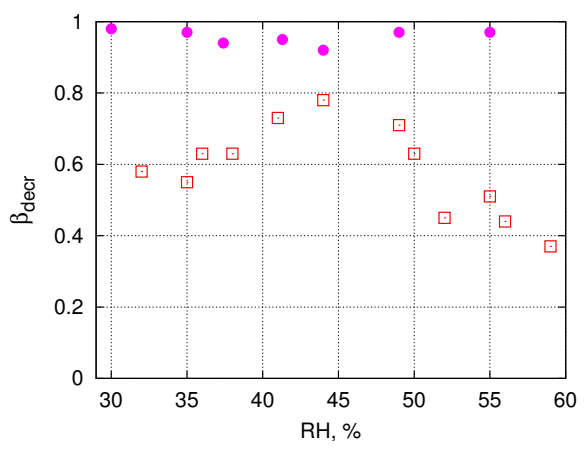

(b)

Figure 4: Humidity dependence of the stretch factors $\beta$ in the process of torque $M_{\omega}$ increase (a) and decrease (b); experimental data are shown with squares, numerical simulation data are shown with circles.

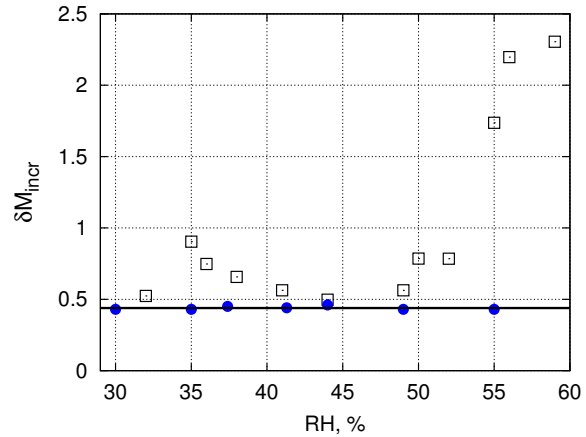

(a)

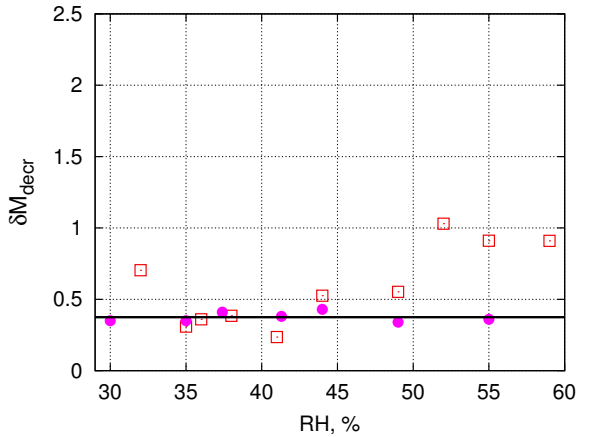

(b)

Figure 5: Humidity dependence of the reduced torque $\delta M_{\text {incr }}$ (a) and $\delta M_{\text {decr }}$ (b); experimental data are shown with squares, numerical simulation data are shown with circles.

charge distribution and $t$ is shifted so that $U_{D C}$ is switched on at $t=0$ and switched off at $t_{o f f}$.

The process shown in Fig. 2 can be described by time constants $\tau_{\text {incr }}, \tau_{\text {decr }}$, stretch factors $\beta_{i n c r}, \beta_{\text {decr }}$, reduced torques $\delta M_{\text {incr }}=\Delta M_{i n c r} / M_{\omega, \text { incr }}(t=0)$ and $\delta M_{\text {decr }}=\Delta M_{\text {decr }} / M_{\omega, \text { incr }}(t=0)$. These parameters plotted as functions of the relative humidity of ambient air are shown in Figs. 3.5 .

\section{Theoretical consideration and discussion}

Possible mechanism of the observed effects can be associated with the charge accumulation on the fused silica plate of the oscillator under the action of the electric field produced by the ESD. Charge migrates along the surface due to the surface conductivity. It is well known that in air the surface conductivity caused 
by adsorption of water vapor is much higher than the internal conductivity of fused silica [16, 17. Silanol groups $(-\mathrm{Si}-\mathrm{OH})$ are formed by chemisorption of water molecules. These polar silanol groups may attract and bind water by physisorption. Electrical conductivity on the $\mathrm{SiO}_{2}$ surface is associated with protons which come from the dissociation of absorbed water molecules. Surface resistivity $\rho_{s}$ as a function of the relative humidity of the surrounding air was measured in [18. The large variation of $\rho_{s}$ with the relative humidity level 135 was explained by conduction due to a hopping of charge carriers between places where the adsorption occurs. The following dependence of the surface resistivity of a fused silica sample on the relative humidity $h$ has been obtained [19]:

$$
\rho_{s}=\rho_{s, 0} \exp (\alpha \sqrt{(1-h) / C h}) .
$$

where $\rho_{s, 0}, \alpha$ and $C$ are some constants.

One can expect that the torque evolution time constant is proportional to the surface resistivity, similarly to the case of bulk conductivity. Fits of $\tau_{\text {incr }}(h)$ and $\tau_{\text {decr }}(h)$ with the functions similar to Eq. 8 are shown with solid lines in Fig. 3. One can see that the fits are good.

In order to interpret theoretically the experimental results a 3D numerical model of the interaction between the fused silica plate and the ESDs has been developed using FEM modeling software. The oscillator's frame and other surroundings are also included in the model. A numerical solution of the Poisson equation is used. The torque $M$ is computed by integration of the Maxwell stress tensor. The dependence of the torque $M$ and the coefficient $A$ in the Eq. 1 on the applied voltage and the distance between the ESDs and the plate 150 has been investigated. The calculated value of $A$ agrees well with the measured value within the limits of measurement error. This model is static, i.e. it does not account for the transport of charge carriers in dielectrics.

In order to simulate the charge dynamics a dynamical model using PoissonNernst-Planck (PNP) equations has been developed. It computes the time dependent response of the oscillator to the electric field produced by the ESD when a step-like voltage is applied to it. This model is based on the following assumptions: positive and negative charges are present only on the surface of the oscillator's plate. The bulk contains no free charges. The amount of charge of each sign is equal, so that the plate as a whole is electrically neutral. The 160 dynamics of the charge carriers and their interaction should satisfy the charge continuity equation and the Poisson equation:

$$
\begin{aligned}
& \frac{\partial n}{\partial t}+\nabla\left(-D^{-} \nabla n+\mu^{-} n \nabla V\right)=0 \\
& \frac{\partial p}{\partial t}+\nabla\left(-D^{+} \nabla p-\mu^{+} p \nabla V\right)=0 \\
& \left.\triangle V\right|_{b u l k}=0 \\
& \left\{\vec{N}\left(-\epsilon_{0} \epsilon_{r, a i r} \vec{\nabla} V+\epsilon_{0} \epsilon_{r, \text { silica }} \vec{\nabla} V\right)\right\}_{\Sigma}=e(p-n),
\end{aligned}
$$


where $p, n$ are the surface densities of positive and negative charge carriers respectively. They have elementary charge $e$, electrical mobilities $\mu^{+}, \mu^{-}$and diffusion coefficients $D^{+,-}=\mu^{+,-} k_{B} T / e$ according to the Nernst-Einstein rela165 tion. $V$ is the electrical potential, $\epsilon_{r, a i r}=1, \epsilon_{r, s i l i c a}=3.9$ are relative permittivities of air and fused silica, $\vec{N}$ is the vector normal to the surface $\Sigma$ of the fused silica plate.

Taking into account that the main charge carriers in absorbed water are suggested to be protons it is assumed that negative ions are stationary and 170 distributed evenly along the surface of the plate (i.e. $\mu^{-}=0, \vec{\nabla} n=0$ ).

Time evolution of electric charge distribution on the surface of the fused silica plate was computed using $2 \mathrm{D}$ geometrical model when the plate was represented by its cross-section perpendicular to the axis of turning. This allows us to drastically speed up the computations. The test computation has shown that the 175 results obtained for 3D and 2D models differ insignificantly. In this numerical model, a small DC offset $\Delta U$ is added to the DC voltage instead of $U_{A C}$ that is applied to the ESD in the experiment.

A range of relative humidity levels $30 \% \leqslant R H<55 \%$ has been taken for the modelling because the significant change of $\delta M_{\text {incr }}$ is observed at $R H>55 \%$ (see Fig. 5a). It can be associated with the dependence of fused silica surface conductivity on the structure of layer of water adsorbed on the surface [20]. The model has two parameters: initial positive charge density $p_{i}\left(n_{i}=p_{i}\right)$ and the electrical mobility of positive ions $\mu^{+}$. Hence at least two experimental quantities need to be selected in order to compare them with numerical results.

185 The time constant $\tau_{i n c r}$ and the reduced torque $\delta M_{\text {incr }}$ are taken for comparison. Within the selected range of the relative humidity levels the values of $\delta M_{\text {incr }}$ and $\delta M_{\text {decr }}$ differ insignificantly. This difference, as well as the difference between $\tau_{\text {incr }}$ and $\tau_{\text {decr }}$ is likely to be omitted in our simplified model. The stretch factors $\beta_{\text {incr,decr }}$ are expected to be close to 1 for this model and also cannot 190 be used as comparison criteria. The calculation shows that $\delta M_{\text {incr }}$ increases with increasing of $p_{i}$ and approaches a constant when $p_{i}>10^{13} \mathrm{~m}^{-2}$. So the constancy of $\delta M_{\text {incr }}$ and equality of the calculated and the measured relaxation time $\tau_{\text {incr }}$ were chosen as a criteria for determining $p_{i}$ and $\mu^{+}$.

We assume that only the charge density $p_{i}$ changes with humidity in this 195 range while the electrical mobility $\mu^{+}$stays constant. This assumption is based on the results of 21]. At $R H=30 \%$ we obtain the fitted value of $\tau_{\text {incr }}=14800 \mathrm{~s}$ (see Fig. 33) if $p_{i}$ is taken to be $5 \cdot 10^{13} \mathrm{~m}^{-2}$ and $\mu^{+}=3.6 \cdot 10^{-13} \mathrm{~m}^{2} /(\mathrm{Vs})$. The values of $p_{i}$ were also computed for several other $\tau_{\text {incr }}$ obtained from the experimental data fit curve (see Fig. 3a, circles). Consequently we obtain that 200 the $p_{i}$ changes from $5 \cdot 10^{13} \mathrm{~m}^{-2}$ to $3.6 \cdot 10^{15} \mathrm{~m}^{-2}$ when the relative humidity changes from $30 \%$ to $55 \%$ and the corresponding surface resistivity values $\rho_{s}=\left(e \mu^{+} p_{i}\right)^{-1}$ are fitted well with Eq. 8 . In Figs. 4.5 the computed values of $\beta_{\text {incr,decr }}$ and $\delta M_{\text {incr,decr }}$ are shown with circles.

The distributions of the surface charge density $P=e(p-n)$ on the plate 205 of the oscillator computed for several time intervals (from $0.1 \tau_{\text {incr }}$ to $\tau_{\text {incr }}$ ) elapsed after the application of DC voltage $U_{D C}=965 \mathrm{~V}$ to the ESD are 


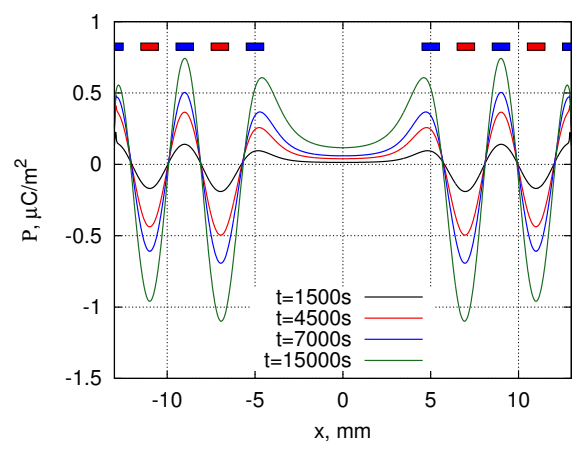

Figure 6: Distributions of surface charge density obtained by numerical simulation for $R H=$ $30 \%$. Distributions are shown for $1500 \mathrm{~s}\left(\approx 0.1 \tau_{\text {incr }}\right), 4500 \mathrm{~s}\left(\approx 0.3 \tau_{\text {incr }}\right), 7000 \mathrm{~s}\left(\approx 0.5 \tau_{\text {incr }}\right)$ and $15000 \mathrm{~s}\left(\approx \tau_{\text {incr }}\right)$. The strips above show positions of the ESD electrodes.

shown in Fig. 6. Such combination of $p_{i}$ and $\mu^{+}$has been chosen so that the calculated time constant corresponds to the experimental one at the relative humidity level $R H=30 \%$. Electric charges redistribute and accumulate on 210 the fused silica plate of the oscillator mirroring the electrodes pattern. The numerical simulation results confirm our initial assumption that the increasing of the torque $M$ is caused by the charge accumulation.

The values of the surface charge carriers density $p_{i}$ and their mobility $\mu^{+}$ were chosen so that the computed value of $\tau_{\text {incr }}$ was close to the measured value. Similar to the case of bulk conductivity, one might expect the following relation to be valid:

$$
\tau_{\text {incr }} \sim G \frac{\epsilon_{0} \epsilon_{r, \text { silica }}}{e p_{i} \mu^{+}}
$$

where $G$ is a dimensional geometrical parameter representing the characteristic length of the system. We have found $G$ to be $\approx 1 \mathrm{~mm}$ in our model.

Note that we can obtain an infinite number of combinations of $p_{i}$ and $\mu^{+}$ that give the calculated $\tau_{\text {incr }}$ equal to the measured one because, in fact, it depends only on the product of these values. This holds true also for other measured values. This indicates the ambiguity of our inverse problem. One can see that $\tau_{\text {decr }}$ and $\beta_{\text {incr, dect }}$ demonstrate only qualitative coincidence between the calculated and the measured values. This is likely due to the simplicity of the model. Nevertheless the model allows one to understand the main aspects of the time dependent interaction between the fused silica plate and electric field of the ESD.

\section{Conclusions}

In this letter, we have investigated the interaction between fused silica plate that is a part of the monolithic fused silica torsion oscillator and the ESD with 
interdigitated electrodes. The time dependent response of the oscillator to a step-like voltage applied to electrodes of the ESD is observed. It is associated with relaxation of the torque of electrostatic force acting on the plate. The time evolution of the torque is calculated from the measured amplitude of the forced oscillations at the selected frequency. The slow increase of the torque with time when DC voltage is applied to the ESD and its slow decrease when the voltage is switched off is observed. The relaxation time of these processes depends on the relative humidity of ambient air and decreases significantly when the humidity increases. It is assumed that the torque relaxation is caused by thistribution of electric charges which is present on th under the action of electric field of the ESD. In order to prove this assumption a numerical model which describes migration of electric charges along the surface of the plate has been developed. The model is based on Poisson-Nernst-Planck equations for the motion of charge carriers which supposedly are protons. They come from the dissociation of absorbed water molecules. It is assumed that the surface charge density changes with humidity and their mobility stays constant. The model allows one to compute the evolution of the charge distribution on the fused silica plate and the change of the torque associated with this evolution. In general, the numerical simulation agrees with the experimental results. Some 250 disagreements can indicate that the proposed simple model does not take into account all aspects of the complex process of the surface conductivity of fused silica associated with adsorbed water such as hopping nature of charge transport. It can be improved. This is a subject for further study.

\section{Acknowledgments}

The authors gratefully acknowledge the support of the Russian Foundation for Basic Research under Grant No. 14-02-00399 and the US National Science Foundation (NSF) and Caltech under Grant No. PHY-1305863. The authors are also grateful to Stan Whitcomb for his invaluable help.

\section{References}

[1] J. J. Ruan, D. Trémouilles, F. Coccetti, N. Nolhier, G. Papaioannou, R. Plana, Reliability assessment of electrostatically driven mems devices: based on a pulse-induced charging technique, Journal of Micromechanics and Microengineering 22 (4) (2012) 045016. doi:10.1088/0960-1317/22/ 4/045016. URL http://stacks .iop.org/0960-1317/22/i=4/a=045016

[2] E. Blokhina, S. Gorreta, D. Lopez, D. Molinero, O. Feely, J. Pons-Nin, M. Dominguez-Pumar, Dielectric charge control in electrostatic MEMS positioners/varactors, Microelectromechanical Systems, Journal of 21 (3) (2012) 559-573. doi:10.1109/JMEMS.2011.2182500. 
270

11

1]

275

[4] C. Affeldt, K. Danzmann, K. L. Dooley, H. Grote, M. Hewitson, S. Hild, J. Hough, J. Leong, H. Lück, M. Prijatelj, S. Rowan, A. Rüdiger, R. Schilling, R. Schnabel, E. Schreiber, B. Sorazu, K. A. Strain,

․ H. Vahlbruch, B. Willke, W. Winkler, H. Wittel, Advanced techniques 280 in GEO 600, Classical and Quantum Gravity 31 (22) (2014) 224002. doi:10.1088/0264-9381/31/22/224002.

URL http://stacks . iop.org/0264-9381/31/i=22/a=224002

[5] J. Aasi, B. Abbott, et al., Advanced LIGO, Classical and Quantum Gravity 32 (7) (2015) 074001. doi:http://dx.doi.org/10.1088/0264-9381/32/ 7/074001.

URL http://stacks .iop.org/0264-9381/32/i=7/a=074001

[6] L. G. Prokhorov, V. P. Mitrofanov, Space charge polarization in fused silica test masses of a gravitational wave detector associated with an electrostatic drive, Classical and Quantum Gravity 27 (22) (2010) 225014. doi:10. 1088/0264-9381/27/22/225014. URL http://stacks .iop.org/0264-9381/27/i=22/a=225014

„ [7] D. Ugolini, C. Fitzgerald, I. Rothbarth, J. Wang, Discharging fused silica optics occluded by an electrostatic drive, Review of Scientific Instruments 85 (3) (2014)-. doi:10.1063/1.4867248.

URL http://scitation.aip.org/content/aip/journal/rsi/85/3/10. $1063 / 1.4867248$

[8] L. Wang, J.-Y. Tang, Q.-A. Huang, Effect of environmental humidity on dielectric charging effect in RF MEMS capacitive switches based on C-V; V properties, Microelectromechanical Systems, Journal of 22 (3) (2013)

300 637-645. doi:10.1109/JMEMS.2012.2237384

[9] D. Molinero, L. Castañer, Modeling and measuring transient discharge current of microelectromechanical switches after dielectric charging by voltage stress, Applied Physics Letters 94 (4) (2009) -. doi:10.1063/1.3075573. URL http://scitation.aip.org/content/aip/journal/apl/94/4/10. $1063 / 1.3075573$

[10] W. Melitz, J. Shen, A. C. Kummel, S. Lee, Kelvin probe force microscopy and its application, Surface Science Reports 66 (1) (2011) 1 - 27. doi:10.1016/j.surfrep.2010.10.001. URL http://ww.sciencedirect.com/science/article/pii/ S0167572910000841 
[11] D. Koptsov, L. Prokhorov, V. Mitrofanov, An interferometric sensor for measuring small oscillations of torsional oscillators Instruments and Experimental Techniques 56 (2) (2013) 215-218. doi:10.1134/ S0020441213010272. URL 10.1134/S0020441213010272

[12] S. Grasso, C. Altucci, et al., Electrostatic systems for fine control of mirror orientation in interferometric GW antennas, Physics Letters A 244 (5) (1998) 360 - 370. doi:10.1016/S0375-9601 (98)00304-1. URL http://www.sciencedirect.com/science/article/pii/ S0375960198003041

[13] M. Hewitson, K. Danzmann, H. Grote, S. Hild, J. Hough, H. Lück, S. Rowan, J. R. Smith, K. A. Strain, B. Willke, Charge measurement and mitigation for the main test masses of the GEO 600 gravitational wave observatory, Classical and Quantum Gravity 24 (24) (2007) 6379.

325 doi:10.1088/0264-9381/24/24/013.

URL http://stacks . iop.org/0264-9381/24/i=24/a=013

[14] J. C. Phillips, Stretched exponential relaxation in molecular and electronic glasses, Reports on Progress in Physics 59 (9) (1996) 1133. doi:10.1088/ 0034-4885/59/9/003.

330 URL http://stacks.iop.org/0034-4885/59/i=9/a=003

[15] U. Zaghloul, B. Bhushan, P. Pons, G. J. Papaioannou, F. Coccetti, R. Plana, On the influence of environment gases, relative humidity and gas purification on dielectric charging/discharging processes in electrostatically driven mems/nems devices, Nanotechnology 22 (3) (2011) 035705. doi:10.1088/0957-4484/22/3/035705.

URL http://stacks . iop.org/0957-4484/22/i=3/a=035705

[16] S.-H. Song, H.-H. Yang, C.-H. Han, S.-D. Ko, S.-H. Lee, J.-B. Yoon, Metal-oxide-semiconductor field effect transistor humidity sensor using surface conductance, Applied Physics Letters 100 (10) (2012) -. doi: $10.1063 / 1.3691936$

(1) URL http://scitation.aip.org/content/aip/journal/apl/100/10/ $10.1063 / 1.3691936$

[17] H. Farahani, R. Wagiran, M. N. Hamidon, Humidity sensors principle, mechanism, and fabrication technologies: A comprehensive review, Sensors 14 (5) (2014) 7881-7939. doi:10.3390/s140507881 URL http://www.mdpi.com/1424-8220/14/5/7881

[18] P. Ho, K. Lehovec, L. Fedotowsky, Charge motion on silicon oxide surfaces, Surface Science 6 (4) $(1967) 440$ - 460. doi: 10.1016/0039-6028(67)90101-X.

350 URL http://www.sciencedirect.com/science/article/pii/ $003960286790101 \mathrm{X}$ 
[19] R. Castagne, P. Hesto, A. Vapaille, Surface conductivity of the insulator of an MIS or MIM device, Thin Solid Films 17 (3) (1973) 253 - 264. doi: 10.1016/0040-6090(73)90134-X. URL http://www.sciencedirect.com/science/article/pii/ $004060907390134 \mathrm{X}$

[20] Y. Awakuni, J. H. Calderwood, Water vapour adsorption and surface conductivity in solids, Journal of Physics D: Applied Physics 5 (5) (1972) 1038. doi:10.1088/0022-3727/5/5/323. URL http://stacks .iop.org/0022-3727/5/i=5/a=323

[21] H. Haspel, N. Laufer, V. Bugris, R. Ambrus, P. Szab[ó]-Révész, Á. Kukovecz, Water-induced charge transport processes in titanate nanowires: An electrodynamic and calorimetric investigation, The Jour-

n nal of Physical Chemistry C 116 (35) (2012) 18999-19009. arXiv:http: //dx.doi.org/10.1021/jp304605k doi:10.1021/jp304605k.

URL http://dx.doi.org/10.1021/jp304605k 